\title{
Die Doppelgesichtigkeit der Sharing Economy Vorschläge zu ihrer gemeinwohlorientierten Regulierung
}

REINHARD LOSKE

Wer sich heute mit der Zukunft der Arbeit beschäftigt - erst recht wenn das aus einer emanzipatorischen Perspektive geschieht -, der kann nicht an der Frage vorbei, wie die an Bedeutung gewinnende „Ökonomie des Teilens" (Sharing oder Share Economy) in ihren verschiedenen Ausprägungen unsere Art, tätig zu sein, zu arbeiten und zu wirtschaften, verändern wird. ${ }^{1}$ Eine Verengung der Debatte zur „Zukunft der Arbeit“ auf die Frage, was denn für die abhängig Beschäftigten nun ,gute Arbeit“ sein soll, oder darauf, wie Digitalisierung und Robotisierung Quantität und Qualität der Erwerbsarbeit beeinflussen, würde zu kurz springen. Wenn sich das Mischungsverhältnis von Erwerbsarbeit, Eigenarbeit und gesellschaftlicher Tätigkeit ändert, von formeller und informeller Ökonomie, von produktiver und reproduktiver Tätigkeit, dann muss die Gesellschaft als Ganze betrachtet werden, nicht nur die Erwerbswelt. Das sollte auch aus gewerkschaftlicher Perspektive gelten.

Hier wird die These vertreten, dass wir bereits auf dem Weg in eine Ökonomie sind, in der Formen kooperativen Wirtschaftens gegenüber rein kompetitiven (wettbewerbsorientierten) Formen an Bedeutung gewinnen werden, was auch Konsequenzen für unsere Formen des Arbeitens hat. Das Teilen, der kollaborative Konsum und die Prosumentennetzwerke, in denen die Entfremdung zwischen Produzierenden und Konsumierenden zu einem guten Teil überwunden werden kann, all das wird an Bedeutung zunehmen. ${ }^{2}$ In diesem Beitrag soll eine Konzentration auf die sogenannte Sharing Economy und die erforderlich werdenden diesbezüglichen Gestaltungs- und Regulierungsmaßnahmen erfolgen.

\section{Sharing Economy: Zugang statt Besitz}

In einem 2010 erschienenen Standardwerk zur Sharing Economy heißt es, sie sei eine Wirtschaftsform, die auf Netzwerken von verbundenen Individuen und Gemeinschaften aufbaue und sich deutlich von der traditionellen Ökonomie unterscheide, die auf zentralisierten Institu- tionen fuße. Durch die Sharing Economy werde die Art $\mathrm{zu}$ produzieren, $\mathrm{zu}$ konsumieren, $\mathrm{zu}$ finanzieren und $\mathrm{zu}$ lernen grundsätzlich transformiert. Gemeinsam sei allen Spielarten des Sharing, dass sie eine kritische Größe überschritten, über freie Ressourcen verfügten, den Gemeinschaftsgedanken pflegten und auf wechselseitiges Vertrauen unter Unbekannten setzten. ${ }^{3}$ Häufig heißt es auch plakativ: „Nutzen statt besitzen!“ oder „Access trumps ownership!“ („Zugang sticht Eigentum!“).

Prominente und gegenwärtig vieldiskutierte Felder der Sharing Economy sind das Car, Ride und Bike Sharing, Fahrgemeinschaften und Nachbarschaftsautos (Verkehr), das Apartment Sharing und Couchsurfing (Wohnungen), das Office Sharing, Häuser der Eigenarbeit und Repair-Cafés (Gewerbeimmobilien und Orte der Zusammenarbeit), der Kleidertausch und die Upcycling-Mode (Kleidung), das Food Sharing, Food Saving und Urban Gardening (Nahrungsmittel), das Co-Working, Co-Making und die Co-Creation (Produktentwicklung), die Maschinenringe und der Werkzeugverleih (Gebrauchsgegenstände), das Crowdfunding, Crowdinvesting und Crowddonating (Finanzierung), das Time Banking (Ehrenamt und wechselseitige Hilfe) sowie das Shared Learning (Bildung). ${ }^{4}$

\footnotetext{
Ich widme diesen Text meinen akademischen Lehrern Elmar Altvater und Hans Christoph Binswanger, die 2018 verstorben sind.

2 Loske, R. (2014a): Neue Formen kooperativen Wirtschaftens als Beitrag zur nachhaltigen Entwicklung, in: Leviathan 42 (3), S. 463-485.

3 Botsman, R./ Rogers, R. (2010): What's mine is yours. How collaborative consumption is changing the way we live, London.

4 Dem geneigten Leser mögen das zu viele Anglizismen sein eine Kritik, die der Autor gut nachvollziehen kann, aber da es sich beim Sharing um eine weltweite Entwicklung handelt, hat sich das Englische auch in der Share oder Sharing Economy als „Fachsprache" durchgesetzt.
} 
In der Sharing Economy gibt es von idealistisch motivierten Gemeinschaften bis zu rein kommerziell agierenden Unternehmen eine enorme Bandbreite an Praktiken und Akteuren, die gesellschaftlich, politisch und ökonomisch unterschiedlich zu bewerten sind. Es ist eben ein gewaltiger Unterschied, ob über Food Sharing, Kleidertausch, Nachbarschaftsautos und städtische Gemeinschaftsgärten geredet wird oder über Großunternehmen wie Uber (Fahrdienstvermittlung), Airbnb (Privatzimmervermittlung), car2go (Autonutzung) oder Helpling (Haushaltsdienstleistungen), über sozial inspirierte kommunale Gemeinwohlinitiativen oder global agierende Vermittlungsplattformen mit Monopolambition.

Häufig wird die These vertreten, dass die Ökonomie des Teilens durch eine Dezentralisierung der Wertschöpfung, eine Steigerung von Sozialkapital, eine Entlastung der Umwelt sowie eine bessere Ausnutzung vorhandener Ressourcen zumindest ein theoretisch hohes Potenzial besitze, zur nachhaltigen Entwicklung im Allgemeinen und zu nachhaltigen Konsumformen und Lebensstilen im Besonderen beizutragen. ${ }^{5}$ Manche Protagonisten der Sharing Economy vertreten gar die These, sie führe über Grenzkosten nahe null für die Bereitstellung von Produkten und Diensten zur sukzessiven Verdrängung kapitalistischer Grundmuster (Eigentumsfixierung, Wettbewerbs- und Wachstumsorientierung, Gewinnerwirtschaftungs- und Akkumulationszwang) durch kooperative Verhaltensweisen, weshalb sich soziale und ökologische Wohlfahrtseffekte quasi wie von selbst einstellten. ${ }^{6}$

Entgegen diesen positiven Zuschreibungen wird aber auch zunehmend darauf hingewiesen, dass mit der Sharing Economy Probleme einhergehen können. So drohten im schlechtesten Fall

- soziale Erosionsprozesse durch Lohndumping, das Unterlaufen sozialstaatlicher Errungenschaften und Arbeitsplatzabbau im formellen und tarifvertraglich und arbeitsrechtlich gesicherten Segment der Volkswirtschaft,

- gesellschaftliche Fehlentwicklungen durch eine Kommerzialisierung des menschlichen Miteinanders und die ökonomische Kolonisierung auch derjenigen Sphären der Gesellschaft, die bislang überwiegend im Mo- dus der sozialen Kooperation und des wechselseitigen Vertrauens gestaltet wurden,

- unfaire Wettbewerbspraktiken durch digitale Vermittlungsmonopole, schlecht bezahlte Arbeit und die ungleiche Behandlung von Gleichem (etwa von Uber-Fahrern und Taxifahrern),

- fragwürdige Ballungen von Wissen und Macht bei den digitalen Plattformbetreibern und daraus resultierend Datenschutzprobleme sowie die Gefährdung von Persönlichkeitsrechten der Plattformnutzer,

- Steuermindereinnahmen des Staates, weil viele Sharing-Aktivitäten sich als Teil einer Schattenökonomie etablieren könnten,

- ökologisch negative Effekte durch die konsumstimulierende Wirkung der multioptionalen Sharing Econo$m y$, die letztlich nicht zu weniger Ressourcenverbrauch und Umweltbelastung führe, sondern zu mehr. ${ }^{7}$

Ob die sozialen und ökologischen Potenziale der Sharing Economy tatsächlich erschlossen werden oder die negativen Effekte letztlich dominieren, hängt vor allem von der konkreten Gestaltung und auch Regulierung der einzelnen Sektoren der Sharing Economy ab. ${ }^{8}$

Bevor nun einige Vorschläge zur adäquaten Gestaltung und Regulierung der Sharing Economy gemacht werden, sollen die gesellschaftlichen Perspektiven auf das Phänomen zunächst genauer unter die Lupe genommen werden. Bislang wurde, auch vom Autor ${ }^{9}$, zumeist lediglich zwischen Sharing-Optimisten und Sharing-Pessimisten unterschieden, wobei erstere eher chancenorientiert argumentieren und viel von Teilhabe, Zugang, Optionserweiterung, Kooperation, Vernetzung und Ressourcenschonung sprechen, während letztere eher auf Probleme wie Sozialdumping, Totalkommerzialisierung, Monopolmacht der Plattformbetreiber, Datenmissbrauch und nicht-nachhaltige Konsumstimulierung hinweisen.

Ein genauerer Blick auf die Diskussions- und Interessenlandschaft führt aber zu dem Ergebnis, dass die bloße Optimismus/Pessimismus-Dichotomie unterkomplex ist und sich mindestens vier Grundperspektiven auf die Sharing Economy identifizieren lassen, die nachfolgend präsentiert werden sollen. Dabei wird so vorgegangen, dass
5 Heinrichs, H. (2013): Sharing economy: A potential new pathway to sustainability, in: Gaia 22 (4), S. 228-231.

6 Vgl. z. B. Rifkin, J. (2014): The zero marginal cost society. The internet of things, the collaborative commons, and the eclipse of capitalism, New York.

7 Für eine Zusammenschau der kritischen Perspektiven vgl. Loske, R. (2014b): Politische Gestaltungsbedarfe in der Ökonomie des Teilens. Eine Betrachtung aus sozial-ökologischer Perspektive, in: ifo-Schnelldienst 27 (21), S. 21-24; Loske, R. (2014C): Aufwachen bitte! Überlasst die Sharing Economy nicht den Internetriesen, in: Die Zeit, Nr. 43 v. 16.10 .2014
8 Loske, R. (2018a): Die Share Economy: Dumpinghölle oder Nachhaltigkeitsparadies?, in: Gesellschaft - Wirtschaft Politik 67 (2), S. 181-187; Loske, R. (2018b): Die Share Economy: Nachhaltigkeitstreiber oder Konsumstimulator?, in: Dörr, J./Goldschmidt, N./Schorkopf, F. (Hrsg.): Die Share Economy. Institutionelle Grundlagen und gesellschaftspolitische Rahmenbedingungen, Tübingen (im Erscheinen).

9 Loske, R. (2015): Sharing Economy. Gutes Teilen, schlechtes Teilen?, in: Blätter für deutsche und internationale Politik 11/2015, S. 89-99. 
die Position zunächst beschrieben und dann auf ihre argumentativen Stärken und Schwächen hin beleuchtet wird jeweils in kurzer Form.

\section{Sharing als Nachhaltigkeitstreiber und Beitrag zur "guten Gesellschaft"}

Position 1 sieht im Sharing vor allem das Potenzial, sozialen Zusammenhalt zu stiften, Teilhabemöglichkeiten zu schaffen sowie Ressourcen effizienter zu nutzen und zu schonen. Hier gilt Sharing als Nachhaltigkeitstreiber und Beitrag zu einer "guten Gesellschaft“. Politische Regulierung soll vor allem dazu beitragen, förderliche Rahmenbedingungen für das Sharing sicherzustellen, zugleich aber dessen negative Nebeneffekte einzudämmen.

Nicht selten wird argumentiert, die gemeinschaftliche Nutzung von Fahr-, Werk- und Spielzeugen, Gebäuden, Geräten und Maschinen, Kleidung, Flächen und Nahrungsmitteln, Wissen und Kompetenzen biete ein enormes Potenzial für Ressourceneinsparung und Umweltentlastung, stifte sozialen Zusammenhalt durch Kooperation und Rückbindung und ersetze egoistische Motive Schritt für Schritt durch eher altruistische. Dem Sharing-Modus, der im gesellschaftlichen Alltag an die Stelle kompetitiver Grundorientierungen trete, wird eine transformative und letztlich systemsprengende Kraft zugeschrieben. Am Horizont erscheint nichts Geringeres als das Ende des Kapitalismus, wie wir ihn kennen. Diese Sichtweise findet auch in ökologisch motivierten Kreisen durchaus erheblichen Zuspruch. ${ }^{10}$

Diejenigen, die dem Sharing-Modus ausschließlich oder überwiegend sozial-ökologisch positive Eigenschaften zuschreiben, haben durchaus gute Argumente und können auf erfolgreiche Praxisbeispiele verweisen. Auch wird von ihnen erkannt, dass ein gewisses Maß an staatlicher Regulierung erforderlich ist, um Mindeststandards (etwa in den Bereichen Umwelt-, Arbeits- und Datenschutz oder Wettbewerbsfairness) sicherzustellen. Eine naiv-euphorische Sicht, wie sie in konsumkritischen, ökologisch und solidarisch orientierten Kreisen noch bis vor kurzem durchaus zu finden war, ist heute einem realistischeren Blick gewichen, der Licht und Schatten gleichermaßen sieht.

Insgesamt wird aber noch immer nicht hinreichend klar gesehen, dass es zum Wesen des modernen Kapitalismus gehört, neue soziale Praktiken, die zunächst nur in Nischen gedeihen und oft altruistisch motiviert sind, als Frischzellenkur zu nutzen und sie in Business Cases zu transformieren oder dies zumindest zu versuchen. ${ }^{11} \mathrm{Im}$ mer wieder strebt der Kapitalismus danach, ökonomisch bislang nicht kolonisierte Sphären des menschlichen Miteinanders zu Geschäftsfeldern zu machen, quasi Gemeinsinn in „Kauf Dich glücklich!“ zu transformieren.

Dieses ubiquitäre Verwertungsstreben, das sozial und ökologisch verheerend wirken kann, in politischen Analysen außer Acht zu lassen ist sträflich, ja naiv, zumal dann, wenn aus diesen Analysen adäquate Gestaltungs- und Regulierungsvorschläge abgeleitet werden sollen.

\section{Sharing als Business Case und Treiber von günstigem „Konsum für alle“}

Position 2 hält das Sharing insbesondere für ein riesiges Geschäftsfeld, in dem sehr viel Geld verdient werden kann. Schaut man sich etwa die Börsenwerte von Unternehmen wie Uber oder Airbnb an, die extrem hoch liegen und in keinem Verhältnis zu den aktuellen Umsatzzahlen und Gewinnen stehen, so wird klar, dass es sich hier vor allem um Zukunftserwartungen handelt.

In der Branche herrscht Goldgräberstimmung, weshalb es auch nicht verwundert, dass man politischen Regulierungen und Restriktionen für den kommerziellen Teil der Sharing Economy mit Ablehnung begegnet. Gern wird zwar vom notwendigen regulatorischen Dialog mit Regierungsstellen gesprochen und die Notwendigkeit „besserer Regulierung“ beschworen , aber schaut man sich die Verlautbarungen der Interessenverbände der Sharing Economy genauer an, so wird die Stoßrichtung schnell klar: Die Politik soll sich zurückhalten und möglichst alles unterlassen, was die Wachstumsaussichten der Branche eintrüben könnte. ${ }^{12}$

Begründet wird das Plädoyer für optimale Marktentwicklung und staatliche Zurückhaltung damit, dass mit einer Kostenreduzierung für Dienstleistungen aller Art auf breiter Front zu rechnen sei. So kämen immer mehr Menschen in den Genuss von Dingen und Diensten, die sie sich ohne Sharing möglicherweise gar nicht leisten könnten. Hierdurch wiederum werde neues Wachstum generiert, das zur Konjunkturbelebung beitrage. Mithin kämen restriktive Sharing-Regulierungen quasi einem unsozialen Akt des Staates gleich, weil sie „den Menschen“ den Zugang zu günstigen und allumfassenden Dienstleistungen vorenthielten und das potentielle Wachstum unnötig schwächten.

Die Aussicht auf eine umfassende „Demokratisierung “ des Konsums („Alles billig für alle!“) durch Sharing ist ein Aspekt, der von den Plattformbetreibern in ihren Werbekampagnen geschickt ins Zentrum gerückt wird. Das öko-

10 Leismann, K. / Schmitt, M. / Rohn, H. / Baedeker, C. (2012): Nutzen statt Besitzen. Auf dem Weg zu einer ressourcenschonenden Konsumkultur, Heinrich-Böll-Stiftung: Kurzstudie Band 27, Berlin, https://www.boell.de/sites/default/files/ Endf_NutzenStattBesitzen_web.pdf

11 Morozov, E. (2014): Don't believe the hype, the 'sharing economy' masks a failing economy, in: The Guardian v. 28.09. 2014

12 EURO Freelancers (2016): Manifesto of the European Sharing Economy Coalition, http://www.eurofreelancers.eu/ european-sharing-economy-coalition 
nomische Interesse an Umsatz- und Renditemaximierung kommt auf Samtpfoten daher und wird gern mit den Idealen von Menschenfreundlichkeit, Freiheit und Optionserweiterung aufgeladen. Kritiker der kommerzialisierten Sharing Economy unterschätzen oft die Potenz dieser Marketingstrategie, die nicht selten verfängt. Fakt ist nämlich, dass viele Menschen in Sachen „Kostenoptimierung“ an einer kognitiven Dissonanz leiden: Als Arbeitnehmer wollen sie sichere Arbeitsplätze und gute Bezahlung, als Unternehmer Gewinnaussichten und ein gewisses Maß an Planungssicherheit, als Bürger funktionierende öffentliche Güter und Sicherheit. Als Konsumenten jedoch wechseln sie schnell in die Position der Schnäppchenjäger und Pfennigfuchser, die sich wenig um die „Produktionsbedingungen“ der konsumierten Güter und Dienstleistungen scheren. Auf diesen Schleier des Nichtwissens oder Nichtwissen-Wollens setzen die Marketing-Strategen der kommerziellen Sharing Economy.

Dieses Verhalten ist menschlich, vielleicht allzu menschlich - und deshalb auch nicht moralisch zu kritisieren. Aber Aufgabe guter Politik im aufklärerischen Sinne ist es sehr wohl, der Öffentlichkeit die Zusammenhänge zu verdeutlichen und klarzumachen, dass die Schnäppchen der einen mit den niedrigen Einkommen der anderen korrespondieren, die exorbitanten Gewinnaussichten der Plattformbetreiber mit dem Druck auf die Realwirtschaft und den Lohnsektor im Zusammenhang stehen, allfällige Strategien des Kostendrückens auf individueller Ebene mit gesellschaftlichen Kosten in Form von Solidaritätsverlusten einhergehen. Kommerziell betriebenes Sharing hat eben auch einen gesellschaftlichen Preis. Den auszublenden ist höchst unpolitisch und in der Konsequenz unsozial.

\section{Sharing als Dumpinghölle und Treiber realwirtschaftlicher Erosion}

Position 3 sieht vor allem die Risiken für die konventionelle Wirtschaft, die sozialen Standards und die (tarifvertraglich abgesicherte) Beschäftigung. Vor übertriebenen Erwartungen in Sachen Zusammenhalt und Nachhaltigkeit wird gewarnt, starke politische Regulierung und auch Eindämmung wird angemahnt.

Der Vorsitzende des Deutschen Gewerkschaftsbundes etwa spricht vom Sharing als "moderner Sklaverei“. ${ }^{13}$ Im „Plattform-Kapitalismus “ ${ }^{14}$ drohe die Erosion sozialstaatlicher Errungenschaften und eine allumfassende De-Solidarisierung der Gesellschaft, also das exakte Gegenteil dessen, was die Sharing-Enthusiasten voraussähen.

Mittelständische Unternehmen, etwa des Taxi- oder Hotelgewerbes, sehen sich durch ungleiche Regulierung in einen ruinösen Wettbewerb getrieben und in ihrer Existenz bedroht. Ihre Interessenverbände streiten in dieser Sache Seit an Seit mit den Gewerkschaften. Von den potenten Wirtschaftsakteuren der Sharing Economy und ihren publizistischen Unterstützern wird diese Konstella- tion gern als Kampf der „Old Economy“ gegen die „New Economy“ dargestellt, als Versuch uneinsichtiger Beharrungskräfte, den Fortschritt aufzuhalten.

Verbraucher- und Datenschützer wiederum verweisen auf die Gefahren mangelnder Sicherheitsstandards, mangelnden Versicherungsschutzes und allzu freigiebigen Umgangs mit persönlichen Daten in der Sharing Econo$m y .{ }^{15}$ Zugleich aber müssen sie zur Kenntnis nehmen, dass immer mehr Menschen von der Möglichkeit des Teilens Gebrauch machen, sich also freiwillig in die schöne neue Welt des Teilens begeben.

Zweifel am Sharing werden zunehmend auch aus ökologischer und konsumkritischer Richtung laut: Zwar sei es richtig, dass Teilen potenziell umweltentlastend und ressourcenschonend wirke, weil theoretisch weniger Güter produziert und gekauft werden müssten. Da das Ganze aber mehr und mehr von einer sozial-ökologisch inspirierten Praxis zu einem wachstumsorientierten Business Case werde, gehe es nicht mehr um Konsumbeschränkung und bessere Ressourcenauslastung, sondern um die Stimulierung von multioptionalem Konsum für jedermann zu jeder Zeit an jedem Ort. Mit Nachhaltigkeit habe das alles rein gar nichts mehr zu tun.

Unzweifelhaft ist: Die Sharing-Kritiker sehen realistische Gefahren. Allerdings insinuieren sie auch, der Status quo sei per se schützenswert, obwohl es auch in der ökonomischen Konstellation unserer Tage höchst fragwürdige Strukturen gibt. So müssen sie sich fragen lassen, ob es nicht Kartelle gibt, denen man durch etwas mehr frischen Wettbewerbswind die ungerechtfertigten Renditen wegnehmen sollte. Und bietet es für die ökologische Gesamtbilanz nicht doch auch Chancen, wenn ehedem nur in der Nische existierende Praktiken auch im Mainstream-Markt umgesetzt werden, wenn Automobilkonzerne sich um Car Sharing kümmern, Baumärkte Werkzeugverleih anbieten, Banken Crowdfunding-Projekte ins Portfolio nehmen? Kurz: Ist es nicht doch ein wenig unterkomplex, die vielfältigen Sharing-Praktiken und -Experimente nur als Bedrohung eines guten Istzustandes durch einen schlechten Geist zu interpretieren?

Gewerkschaften sollten eine differenziertere Haltung zur Sharing Economy beziehen, als sich lediglich eins zu

13 DGB warnt vor neuen Modellen der Ausbeutung, in: Spiegel online v. 17. 08. 2014, http://www.spiegel.de/wirtschaft/ soziales/uber-und-andere-dgb-warnt-vor-moderner-sklave rei-a-986545.html

14 Lobo, S. (2014): Auf dem Weg in die Dumpinghölle, in Spiegel online v. 03. 09. 2014, http://www.spiegel.de/netzwelt/ netzpolitik/sascha-lobo-sharing-economy-wie-bei-uber-istplattform-kapitalismus-a-989584.html

15 Verbraucherschützer warnen vor der Sharing Economy, in: Wirtschaftswoche, WiWo-Gründer-Newsletter v. 29. 06. 2015, http://gruender.wiwo.de/warum-verbraucherschuetzer-vor-der-sharing-economy-warnen 
eins mit ständischen Interessen zu verbinden und eine bloße Abwehrhaltung einzunehmen. Es geht vielmehr darum, eine klare Position zu der Frage zu entwickeln, wo die Sharing Economy wirklich auf der Basis von echtem Teilen funktioniert und deshalb grundsätzlich unterstützungswert ist und wo sie auf reiner Profitorientierung oder gar der Umgehung rechtlicher Bestimmungen basiert und deshalb strengeren Regeln unterworfen werden muss. Intelligente Gewerkschaftspolitik mit ihrem reichen Erfahrungsschatz muss diese Scheidelinie ziehen können.

\section{Sharing als Treiber einer neuen Unverbindlichkeit und Eigentumsentwertung}

Position 4 lässt sich am ehesten als sozial-wertkonservativ beschreiben und betont, dass das Sharing-Wachstum und der Bedeutungsverlust des Eigentums zu einer neuen Unverbindlichkeit führten, weil niemand mehr wirklich dauerhafte Verantwortung für die Dinge und die Gemeinschaft übernehmen wolle, nur im Augenblick lebe und kein „soziales Kapital“ aufbaue.

Die These lautet im Grunde, dass das Sharing nur ein weiteres Indiz für den Eintritt in das Zeitalter der sozialen Unverbindlichkeit sei, in dem immer weniger Menschen bereit sind, sich dauerhaft an andere Menschen und an langlebige Dinge zu binden. Wer zu seinen Kolleginnen oder Kollegen mittags „Mahlzeit!“, abends „Schönen Feierabend!“, freitags „Schönes Wochenende!“ und jeden Sommer wieder „Schönen Urlaub!“ sagen könne, der empfinde eine ganz andere Verantwortung für das Miteinander in der Arbeitswelt als der „Freelancer", der gerade an seinem neuesten „Projekt" werkele und nur ab und an mal ein paar unverbindliche Worte mit Zufallsbekanntschaften im „Co-Working Space“ wechsle.

Wer sich auf eine zeitlich (prinzipiell) unbefristete Beziehung (mit Kindern) oder auf Wohneigentum einlasse, der empfinde eine ganz andere Verantwortung für das Funktionieren der Gesellschaft als all die Lebensabschnittspartner und Couchsurfer, die überwiegend im Hier und Jetzt lebten. Und wer seinen eigenen Garten nutze, gestalte, hege und pflege, der wirke doch viel nachhaltiger als jeder Urban Gardener, der im nächsten Jahr schon wieder fortziehen könne. Kurz: Sozialkapital und Verantwortung entstünden nur durch dauerhafte Bindung an Menschen und Dinge, wo aber die Orientierung am jeweils nächsten Hype vorherrsche, gedeihe nichts dergleichen.

Diese Argumentationsfigur enthält in jedem Fall nachvollziehbare Elemente. So ist der „Sharing Lifestyle“ mit seinen extrem hohen Anteilen an Mobilität, Flexibilität und Unverbindlichkeit natürlich kein universelles Lebensstil-Modell für alle Menschen in allen Regionen und Altersklassen. Über den „Typ Mensch“, der schon heute an Sharing-Aktivitäten partizipiert, liegen verschiedene Studienergebnisse vor. Zwar stehen Pauschalisierungen immer in der Gefahr, zu stark zu vereinfachen, aber als grob zutreffend darf doch die folgende Aussage gelten:
Die Sharing Community ist überwiegend jung, urban, gut gebildet und experimentierfreudig. ${ }^{16}$ Das Einkommen ist oft (noch) nicht sehr hoch oder sogar niedrig, dürfte aber aufgrund der im Regelfall guten Ausbildung in Zukunft steigen.

Einen Sharing-Totalitarismus, wie er der Gesellschaft von manchen Trendscouts anempfohlen wird, wird es sicher nicht geben. Diese Befürchtung ist nicht begründet, auch wenn das Sharing vor allem in den Großstädten weiter stark an Bedeutung gewinnen wird. Überdies findet in der hier beschriebenen Perspektive doch eine arg idealisierende Betrachtung des Eigentums statt. Sicher ist es, im Guten wie im Schlechten, ein „Bindungsverstärker“. Aber dass Eigentum quasi automatisch auch Verantwortungsgefühl für die Gemeinschaft stiftet, ist doch eine sehr heroische Annahme. Es gibt zu viele Beispiele dafür, dass das nicht zwingend der Fall sein muss. Und es ist sicher kein Zufall, dass die Verfassungen der allermeisten Staaten die Festlegung treffen, dass Eigentum dem Gemeinwohl verpflichtet sein muss.

Auch die sozial-konservative Idealisierung der traditionellen Erwerbsarbeit ist doch etwas einseitig. Natürlich gilt die Tatsache, dass ein gesichertes und reguläres Beschäftigungsverhältnis den meisten Menschen Planungssicherheit für ihr Leben gibt, was oft auch die Voraussetzung für stabiles gesellschaftliches Engagement ist. Deshalb ist es gerade aus sozial-ökologischer Perspektive so wichtig, Erwerbsarbeit für alle anzustreben - in Vollzeit oder, wenn gewünscht, mit reduzierter Arbeitszeit. Aber das heißt doch nicht, dass kollaborative Produktions-, Arbeits- und Konsumtionsformen keinen Eingang in die Wirtschaftswelt finden sollten. Im Gegenteil: Job Sharing, Co-Working, Co-Making oder Co-Creation sind innovative Konzepte, die das Arbeitsleben insgesamt für alle Beteiligten interessanter machen können, auch wenn vor blauäugigen Idealisierungen zu warnen ist.

\section{Politische Gestaltungs- und Regulierungsbedarfe in der Sharing Economy}

Die beschriebenen Grundsichten auf die Sharing Economy mögen vereinfachend sein und Überlappungen aufweisen. Sie spiegeln nach Einschätzung des Autors aber die in der Gesellschaft zu findenden Positionen angemessen wider. Vor allem ist die realistische Einschätzung der Sharing-Konfliktlandschaft eine wichtige Voraussetzung für gute Gestaltung und gute Regulierung dieser noch jungen Form des Wirtschaftens.

16 Institut für Ökologische Wirtschaftsforschung (2016): Befragung: Jeder Dritte ist bereit, Dinge übers Internet mit anderen zu teilen, Pressemitteilung v. 16.06. 2016, https://www. ioew.de/presse/pressemitteilungen/befragung-jeder-dritteist-bereit-dinge-uebers-internet-mit-anderen-zu-teilen 
Wie könnte eine an Gemeinwohlinteressen orientierte Gestaltung und Regulierung der Sharing Economy in ihren Umrissen nun aussehen? Ich meine, es sind vier Gestaltungskreise, die von der Politik anzugehen sind: Definitionsarbeit, Förderung des sozialen Sharing, klare Regulierung des kommerziellen Sharing, Einbettung der Sharing-Gestaltung in das gesellschaftspolitische Leitbild einer nachhaltigen Entwicklung.

Gestaltungskreis 1: Definitorische Klärungen - Was nottut, ist eine realitätsnahe und praxisorientierte Differenzierung. Es gilt, den eher gemeinwohlorientiert arbeitenden vom eher gewinnorientiert arbeitenden Teil der Sharing Economy definitorisch zu scheiden. Nur so vermeidet man, alles in einen Korb zu werfen, und stellt sicher, dass Gleiches gleich und Ungleiches ungleich behandelt wird.

Ein Weg zur Definition der Sharing Economy geht etwa über das Prinzip der Abgrenzung zu anderen konventionellen Business-to-Consumer-Aktivitäten (B2C), etwa zum Leasing, zu Second-Hand-Märkten und zur Leistungserbringung von Privaten für Private in der sogenannten On-Demand Economy, z.B. für Haushaltsdienstleistungen. ${ }^{17}$ Wo Sharing zwar im sympathischen Gewande des Gemeinwohls daherkommt, aber faktisch kommerzielle Dienstleistungen anbietet, kann es gegenüber anderen Segmenten der wettbewerbsorientierten Wirtschaft nicht mit einer Sonderbehandlung rechnen.

Oft ist bereits an der Rechtsform erkennbar, ob sich eine Sharing-Aktivität eher an gemeinnützigen oder eher an kommerziellen Zielen ausrichtet. In der ersten Kategorie überwiegen zumeist Vereine, Stiftungen, Genossenschaften, gemeinnützige GmbHs oder kommunale Eigenbetriebe, in der zweiten Kategorie eher Personen- und vor allem Kapitalgesellschaften. In der ersten Kategorie sind die Akteure meist eher klein und agieren ziel-, nicht wachstumsgetrieben, in der zweiten Kategorie sind sie eher groß und stark wachstumsorientiert. Sind die definitorischen Klärungen erst einmal vorgenommen, gilt es für beide Systeme angemessene Gestaltungs- bzw. Regulierungsregime zu erarbeiten. Ziel muss es dabei sein, einen lernenden Ordnungsrahmen zu schaffen, der Richtungssicherheit im Sinne von Gemeinwohlorientierung, Nachhaltigkeit und Wettbewerbsfairness garantiert, aber auch offen genug ist, um reflexiv auf technische und soziale Innovationen sowie eventuelle Überraschungseffekte reagieren zu können.

Gestaltungskreis 2: Förderung des sozialen Sharing - Wo Sharing gemeinwohlorientiert organisiert ist, hat Politik die Aufgabe, dieses zu fördern, zu stabilisieren und auch vor feindlichen Übernahmen zu schützen, um es im sozialen Modus zu halten. Hier liegen besonders für Städte und Gemeinden zahllose Handlungsmöglichkeiten, vor allem in der Überführung von spontanen Entwicklungen in tragfähige und robuste Strukturen.

Es existiert inzwischen ein weltweites Netz an Sharing Cities, die jeweils ganz eigene Wege zur Förderung der Ökonomie des Teilens gehen: von Seoul über Hamburg bis Toronto. In Amsterdam etwa setzt die Stadtverwaltung gezielt auf die Förderung lokal orientierter Apps, um den globalen Plattformen eine soziale Alternative gegenüber zu stellen; sie hat zu diesem Zweck einen Sharing-Aktionsplan verabschiedet, der Innovation, soziale Teilhabe, Unternehmungsgeist und Nachhaltigkeit in der Stadt befördern soll.

In den verschiedenen Feldern des gemeinwohlorientierten Teils der Share Economy hat man es politisch im Regelfall mit einem hohen Maß an Idealismus auf fast allen Seiten zu tun. Der kritische Faktor ist hier meist die Nachhaltigkeit, verstanden als Dauerhaftigkeit des Engagements der Akteure. Oft hängt der Erfolg solcher Projekte an der Einsatzbereitschaft einer kleinen Gruppe von besonders aktiven Menschen, weshalb ein gewisses Maß an Professionalisierung und öffentlicher Unterstützung auf Dauer unerlässlich ist. Auch arbeitsmarktpolitische Instrumente können hier genutzt werden, um dauerhaft tragfähige Strukturen zu fördern.

Gestaltungskreis 3: Kommerzielles Sharing angemessen regulieren - Dort, wo Sharing eine gewinnorientierte Wirtschaftsaktivität ist, also keine sozial motivierte Interaktion, sondern ein schlichtes Geschäftsmodell, ist eine adäquate Regulierung erforderlich, die Arbeitnehmer-, Verbraucher- und Umweltschutz, Wettbewerbsfairness und die Fiskalinteressen des Staates gleichermaßen im Auge hat.

Bei dieser Regulierungsaufgabe lassen sich wiederum spezifische Teilaufgaben identifizieren. Es geht zum einen um die Regulierung der Basisinfrastruktur für die Share Economy, die Plattformen und Suchmaschinen. Hier muss staatlicherseits systematisch Monopolbekämpfung und, wo erforderlich, auch Entflechtung betrieben werden, um marktbeherrschende Stellungen einzelner Unternehmen zu begrenzen. ${ }^{18}$

Eine weitere Aufgabe der Politik besteht darin, konkrete Grenzen zu ziehen, jenseits derer kommerzielles Sharing als ganz normale Gewerbeaktivität zu gelten hat. Derzeit sind beispielsweise immer mehr Städte dabei, die Anzahl der Tage pro Jahr zu begrenzen, an denen Privatwohnungen (z. B. über Airbnb) vermietet werden dürfen, um steigende Mieten, den Zerfall von Nachbarschaften oder zunehmende Gentrifizierung zu verhindern. Weitere Regulierungsaufgaben liegen etwa in der Sicherstellung von Gewährleistungspflichten (z. B. bei der Personenmitnahme), von Sicherheits- und Hygienestandards (z. B. bei der Zimmervermietung) oder angemessenem Versicherungsschutz (z. B. bei gemeinschaftlicher Gerätenutzung).

17 Frenken, K./ Meelen, T./ Arets, M. / van de Glind, P. (2015): Smarter regulation for the sharing economy, in: The Guardian v. 20.05. 2015

18 Mason, P. (2018): Zerschlagt die digitalen Monopole, in Blätter für deutsche und internationale Politik 2/2018, S. 73-82. 
Im genannten Kontext kommt den Gewerkschaften eine Doppelrolle zu. Sie müssen als „watchdog“ darauf achten, dass Gleiches auch tatsächlich gleich behandelt wird, also Versuche der Unterhöhlung arbeitsrechtlicher und tarifvertraglich vereinbarter Standards skandalisieren und attackieren. Zugleich sollten sie aber bestrebt sein, auch den in der kommerziell ausgerichteten Share Economy beschäftigten Menschen eine gute Interessenvertretung zu werden, auch wenn die Organisation dieser diversen und volatilen Interessen ungleich schwerer fallen dürfte, als dies in Betrieben und Verwaltungen der Fall ist.

Gestaltungskreis 4: Die Einbettung der Sharing Economy in ein nachhaltiges Gesellschaftsmodell - Die Sharing Economy weist derzeit eine hohe Dynamik auf, was auf gesellschaftlichen Wertewandel („Nutzen statt besitzen“, „Befreiung vom Wohlstandsballast"), technische Innovationen (Digitalisierung) und neue Geschäftsmodelle (Service Economy) gleichermaßen zurückzuführen ist. In welche Richtung sie sich weiterentwickelt, in welchem Mischungsverhältnis sich zukünftig kompetitives und kooperatives Wirtschaften darstellen, ist noch nicht ausgemacht. Es kann in Richtung Totalkommerzialisierung laufen, wenn das Feld regellos den wachstumsfixierten Digitalgiganten überlassen wird. Das wäre sozial wie öko- logisch verheerend. Sharing kann aber auch einen Beitrag zur nachhaltigen Entwicklung leisten, wenn es gelingt, die Tendenz zur gemeinschaftlichen Nutzung von Gütern und Diensten überwiegend im sozial-ökologischen Modus zu halten. Das passiert aber nicht von selbst, sondern braucht politischen Willen.

Entscheidend bei der Regulierung der Sharing Economy sind letztlich die Grundmotive, Grundhaltungen und Grundwerte, mit denen sie angegangen wird. Zu bewerten sein wird jede Art des Wirtschaftens, auch die Sharing Economy, letztlich vor dem Hintergrund der Frage, ob sie zur Einhaltung ökologischer Grenzen, zum gesellschaftlichen Zusammenhalt und zur Gerechtigkeit beiträgt.

\section{AUTOR}

REINHARD LOSKE, Dr., ist Professor für Nachhaltigkeit und Transformationsdynamik an der Fakultät für Kulturreflexion der Universität Witten/Herdecke. Arbeitsschwerpunkte: Fragen der sozial-ökologischen Transformation des Wirtschafts- und Gesellschaftssystems, Beratung von Regierungen und Nichtregierungsorganisationen in Europa, Afrika und Südamerika.

reinhard.loske@uni-wh.de 